ARTICLE

https://doi.org/10.1038/s41467-019-09390-9

\title{
A new type of DNA phosphorothioation-based antiviral system in archaea
}

Lei Xiong ${ }^{1,2,3}$, Siyi Liu ${ }^{1,3}$, Si Chen ${ }^{4}$, Yao Xiao ${ }^{1,3}$, Bochen Zhu ${ }^{1,3}$, Yali Gao ${ }^{1,3}$, Yujing Zhang ${ }^{1,3}$, Beibei Chen ${ }^{5}$, Jie Luo ${ }^{2}$, Zixin Deng ${ }^{1}$, Xiangdong Chen $^{5}$, Lianrong Wang (1) ${ }^{1,2,3} \&$ Shi Chen (1) ${ }^{1,2,3}$

Archaea and Bacteria have evolved different defence strategies that target virtually all steps of the viral life cycle. The diversified virion morphotypes and genome contents of archaeal viruses result in a highly complex array of archaea-virus interactions. However, our understanding of archaeal antiviral activities lags far behind our knowledges of those in bacteria. Here we report a new archaeal defence system that involves DndCDEA-specific DNA phosphorothioate (PT) modification and the PbeABCD-mediated halt of virus propagation via inhibition of DNA replication. In contrast to the breakage of invasive DNA by DndFGH in bacteria, DndCDEA-PbeABCD does not degrade or cleave viral DNA. The PbeABCDmediated PT defence system is widespread and exhibits extensive interdomain and intradomain gene transfer events. Our results suggest that DndCDEA-PbeABCD is a new type of PT-based virus resistance system, expanding the known arsenal of defence systems as well as our understanding of host-virus interactions.

\footnotetext{
${ }^{1}$ Key Laboratory of Combinatorial Biosynthesis and Drug Discovery Ministry of Education, School of Pharmaceutical Sciences, Wuhan University, 430071 Wuhan, China. ${ }^{2}$ Taihe Hospital, Hubei University of Medicine, 442000 Shiyan, Hubei, China. ${ }^{3}$ Brain Center, Zhongnan Hospital, Wuhan University, 430071 Wuhan, China. ${ }^{4}$ School of Biology and Pharmaceutical Engineering, Wuhan Polytechnic University, 430023 Wuhan, China. ${ }^{5}$ College of Life Sciences, Wuhan University, 430071 Wuhan, China. These authors contributed equally: Lei Xiong, Siyi Liu, Si Chen, Yao Xiao. Correspondence and requests for materials should be addressed to S.C. (email: shichen@whu.edu.cn)
} 
$\mathrm{V}$ iruses are the most abundant biological entities in the biosphere and are estimated to outnumber their bacterial and archaeal hosts by tenfold. The constant threat of virus predation has led to the evolution of diverse defence mechanisms that target nearly every stage of the viral infectious cycle. These mechanisms involve inhibiting adsorption, blocking viral DNA injection, restriction-modification (R-M), limiting phage growth, toxin-antitoxin systems, abortive infection and CRISPR/Cas systems, as well as the newly discovered prokaryotic Argonaute, BREX, DISARM, Zorya and Dnd defence systems ${ }^{1-6}$.

Among these mechanisms, the use of R-M systems is the most prevalent and best-characterised antiviral approach among Bacteria and Archaea. Generally, R-M systems consist of two contrasting enzymatic activities: (1) a methyltransferase (MTase) that catalyses the transfer of a methyl group to DNA nucleobases within a particular sequence motif of 'self DNA and (2) a restriction endonuclease (REase) cognate that identifies and destroys invading foreign DNA that harbours the same DNA motif when not methylated. Four major types of R-M systems (I, II, III and IV) are classified based on their subunit composition, cofactor requirements, sequence recognition and cleavage mechanisms $^{7}$. Type I R-M systems comprise three subunits, R (restriction), M (modification) and $\mathrm{S}$ (specificity), and the resulting complex binds to a bipartite sequence and requires ATP hydrolysis to cleave at a distant site following DNA translocations $^{8}$. Type II systems are the simplest, which contain separate MTase and REase enzymes to act independently. Type III systems are multifunctional oligomeric proteins consisting of a Mod and a Res subunit. The Mod subunit alone can function independently as a MTase to recognise non-palindromic sequences, only one strand of which can be methylated, whereas the Res subunit needs to pair with the Mod subunit for restriction activity ${ }^{9}$. Type IV systems have no methylase activity and type IV REases recognise and cleave only modified DNA ${ }^{10-12}$. Although type I-III R-M systems are tremendously diverse, the associated DNA modifications most commonly occur in the form of methylation of nucleobase moieties, such as $\mathrm{N}^{6}$-methyl-adenine, $\mathrm{N}^{4}$-methylcytosine and $\mathrm{C}^{5}$-methyl-cytosine.

The Dnd defence system has recently been recognised as a new component of the bacterial innate immune system, in which phosphorothioate (PT) modification is used as a marker to distinguish between self and non-self DNA, analogous to methylation-based R-M systems ${ }^{13,14}$. In contrast to DNA methylation at nucleobases, PT modification occurs on the DNA sugar-phosphate backbone, where replacement of the nonbridging oxygen by sulphur confers nuclease resistance at the PT linkage ${ }^{13,15}$. The Dnd system is organised into three parts: (1) DndACDE proteins form a complex and act as the modification component to catalyse DNA PT modification in a sequenceselective manner ${ }^{16,17}$; (2) DndB is a transcriptional repressor capable of regulating expression of the $d n d B C D E$ cluster and the resulting PT level ${ }^{18}$ and (3) DndFGH proteins function as the R component to recognise and destroy non-PT-modified foreign $\mathrm{DNA}^{14,19}$. All three $d n d F G H$ genes are essential to distinguish and restrict the transformation of non-PT-modified incoming plasmid DNA in Salmonella enterica ${ }^{4}$. Once PT modification is abolished, $S$. enterica suffers double-stranded DNA damage from the unrestrained restriction activity of DndFGH, which consequently leads to growth defects and triggers the SOS response ${ }^{14,20}$. Although the enzymatic activities and mechanism of action of DndFGH is currently unclear, these features resemble the well-characterised 'self/non-self discrimination' strategy of methylation-based R-M systems.

To prevent REase attack on resident DNA, the recognition motifs are generally nearly completely methylated by the MTase counterpart. However, this situation is not observed for Dnd defence systems. Indeed, only 4855 of 40,701 complementary 5'GAAC-3'/5'-GTTC-3' consensus sequences found in the Escherichia coli B7A genome are PT modified to $5^{\prime}-\mathrm{G}_{\mathrm{PS}} \mathrm{AAC}-\mathrm{3}^{\prime} / 5^{\prime}$ G $_{P S}$ TTC-3' (PS: phosphate-sulphur linkage), even in the presence of active DndFGH, ruling out the known R-M mechanisms.

Although the number of described archaeal viruses accounts for only $1 \%$ of all reported prokaryotic viruses, the archaeal viruses exhibit enormously diversified morphotypes and genome contents $^{21-24}$. Many of the unique morphologies of archaeal viruses, including coil-shaped, bottle-shaped, spherical and droplet-shaped with beard-like fibres, have not been observed among bacterial or eukaryotic viruses ${ }^{21}$. Consistent with the uniqueness of viral morphotypes, the genome contents of these archaeal viruses are distinctive, with $\sim 75 \%$ of genes encoding functionally unknown protein $s^{25}$. Although the current knowledge of archaeal viruses has merely touched the tip of the iceberg, one may imagine the development of diverse defence systems during the coevolutionary arms race between archaea and viruses given the distinct morphological and genomic properties of viruses.

Indeed, as in Bacteria, an astonishing array of defence systems have developed among Archaea to restrict invasive viruses and plasmids. For example, $96 \%$ of the sequenced bacterial genomes harbour R-M systems, while 384 (98\%) of the 390 currently available archaeal genomes encode R-M systems (http://rebase. neb.com/rebase/rebase.html) ${ }^{26}$, as of the time of writing of this manuscript. However, only a few archaeal R-M systems have been investigated to date, including R.PabI from the hyperthermophilic Pyrococcus abyssi, R.PspGI from Pyrococcus sp. strain GI-H, R. Sual from the thermoacidophilic Sulfolobus acidocaldarius and their cognate MTases ${ }^{27-30}$. Moreover, archaea wrap their DNA into histone-DNA complexes with the same geometry as DNA in the eukaryotic nucleosomes ${ }^{31}$, prompting us to investigate the aspects of PT modification and PT-related functions in Archaea.

In this study, we first identify PT modifications in halophilic and methanogenic archaea that show diverse sequence specificities and abundance comparable to those observed in bacteria. However, no trace of the restriction component DndFGH is detected in any of the archaeal strains assessed. Instead, we report that PT-modification genes, such as dndCDEA in the halophilic archaeon Haloterrigena jeotgali A29, can defend against viral attack together with pbeABCD, a conserved 4-gene cassette sharing no sequence homology with DndFGH. Moreover, the defence mechanism involves the DndCDEA-mediated PT modification of the self-DNA sequence and the use of PbeABCD to halt virus propagation by inhibiting DNA replication in a PTdependent manner. Unlike the DndFGH-mediated defence mechanism, DndCDEA-PbeABCD does not function through the degradation or cleavage of viral DNA, highlighting a novel mode of action that is distinct from R-M activity and expanding the arsenal of known defence systems, as well as our understanding of host-virus interactions.

\section{Results}

Occurrence of DNA PT modifications in Archaea. Although our understanding of $d$ nd systems and PT modifications in Bacteria has dramatically expanded in the past decade, PT modification and its physiological relevance have not been characterised in Archaea, which comprise up to $20 \%$ of the total biomass on Earth ${ }^{21}$. In this study, we first identified DNA PT modifications in five archaea with homologous PT-modification genes. Based on mass spectral analysis, nuclease-resistant PTlinked dinucleotides were produced upon enzymatic hydrolysis of archaeal DNA (Fig. 1 and Supplementary Fig. 1). The following strains were observed to harbour PT-modified $d\left(\mathrm{G}_{\mathrm{PS}} \mathrm{A}\right)$ : three 


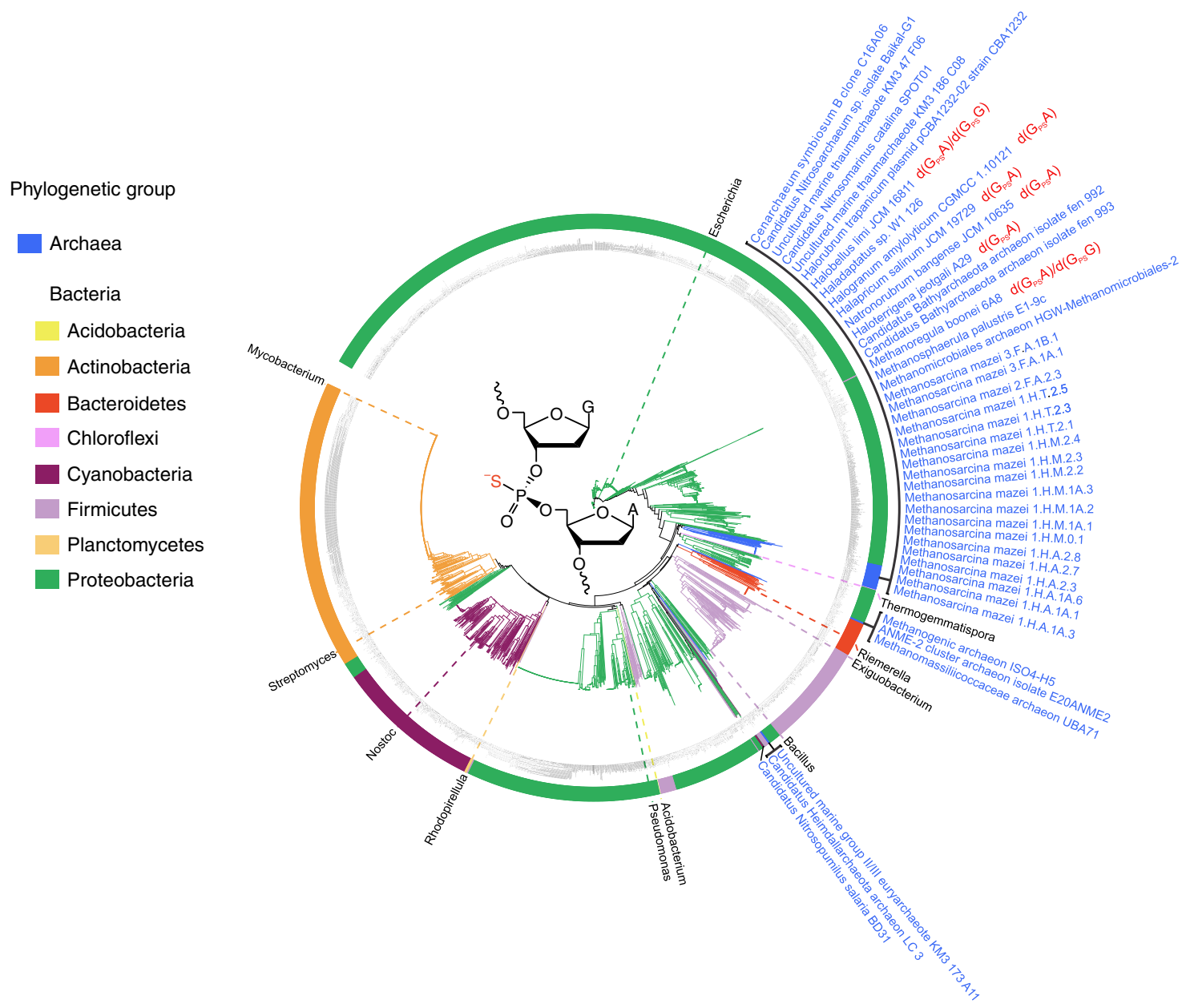

Fig. 1 Maximum likelihood phylogeny of 2642 homologues of the $H$. jeotgali A29 DndCD identified in Archaea and Bacteria. The strain names of 42 archaea, as well as the phosphorothioate (PT) sequence contexts identified in this study are displayed as noted. Each prokaryotic phylum is represented in a distinct colour (see the legend). Bacterial strain names are also provided in Supplementary Data 1. For clarity, at least one familiar genus per colour segment were labelled. The structure of PT-modified $d\left(G_{\mathrm{PS}} \mathrm{A}\right)$ in the $R_{\mathrm{P}}$ configuration is shown in the centre of the tree. Source data are provided as a Source Data file

halophilic archaeal strains, H. jeotgali A29, isolated from saltfermented shrimp jeotgal, and Halapricum salinum JCM 19729 and Halobellus limi JCM 16811, isolated from solar salterns in Korea and China, respectively; and one haloalkaliphilic archaeal strain, Natronorubrum bangense JCM 10635, isolated from a soda lake in Tibet. H. limi JCM 16811 also possesses a detectable level of $d\left(\mathrm{G}_{\mathrm{PS}} \mathrm{G}\right)$. An acidophilic methanogen, Methanoregula boonei $6 \mathrm{~A} 8$, isolated from an acid peat bog in the United States, also shows PT modification at $d\left(\mathrm{G}_{\mathrm{PS}} \mathrm{A}\right)$ and $d\left(\mathrm{G}_{\mathrm{PS}} \mathrm{G}\right)$ of the same order of magnitude (Table 1). These findings reveal the diversity of PT consensus sequences among Archaea. Next, we determined that the dndCDEA operon of $H$. jeotgali A29 was capable of conferring $d\left(\mathrm{G}_{\mathrm{PS}} \mathrm{A}\right)$ modification upon transfer to a new host, Natrinema sp. CJ7-F, an extreme haloarchaeon without endogenous $d n d$ genes (Supplementary Fig. 1).

We next used the DndCD protein sequences (DndE is too small to be annotated in some genomes) of $H$. jeotgali A29 as a query to explore the occurrence and diversity of Dnd systems across archaeal genomes. Using BLAST, we searched the homologues of DndCD in the NCBI databases of nonredundant nucleotide collections (nt), RefSeq Genome (refseq_genomes), whole-genome shotgun contigs (WGS), highthroughput genomic sequences (HTGS), reference genomic sequences (refseq_genomic), and genomic survey sequences (GSS). We filtered the results based on an $e$-value of $\leq 10^{-10}$ and discarded BLAST hits with aligned lengths of $\leq 30 \%$ of the query proteins. A total of 2642 occurrences of $d n d C D$ were identified nested within 2600 bacterial and 42 archaeal genomes (Fig. 1 and Supplementary Data 1). The 42 Archaea harbouring DndCD homologues span the phylogenetic diversity of archaeal lineages, including Methanomicrobia (23), Haloarchaea (7), Thaumarchaeota (6), Bathyarchaeota (2), Thermoplasmata (2), and Heimdallarchaeota (1), as well as an uncultured marine group II/III euryarchaeote KM3_173_A11 (Supplementary Data 1). According to the phylogenetic tree of the 2642 DndCD proteins (archaeal and bacterial DndCD proteins), the archaeal strains do not phylogenetically cluster together but instead are grouped with bacteria, suggesting potential interdomain HGT of Dnd systems between Archaea and Bacteria (Fig. 1).

Genome-wide PT features in Archaea. In contrast to the wellcharacterised $d n d B C D E$ cluster in bacterial genomes, dndCDEA clusters in H. jeotgali A29, N. bangense JCM 10635, H. limi JCM 16811 and $H$. salinum JCM 19729 are not accompanied by $d n d B$, which encodes a negative regulator that binds to the promoter region of the $d n d B C D E$ operon. Upon relief of DndB repression, the transcription of $d n d B C D E$ and the resulting PT frequency increased by 15 - and 2 -fold, respectively, in the bacterial strain $S$. enterica serovar Cerro $87^{18}$. However, in the four haloarchaeal strains, we observed $d\left(\mathrm{G}_{\mathrm{PS}} \mathrm{A}\right)$ at an average frequency of $\sim 2-3 \mathrm{PT}$ 
Table 1 DNA phosphorothioate (PT) modifications in archaeal strains

\begin{tabular}{|c|c|c|c|c|}
\hline Strains & GenBank accession no. & $\begin{array}{l}d\left(G_{p S} A\right) \\
\left(\text { per } 10^{4} n t\right)\end{array}$ & $d\left(G_{P S} G\right)$ & Total PT \\
\hline H. jeotgali A29 & СР031298-СР031304 & $2.8 \pm 0$ & NA & $2.8 \pm 0$ \\
\hline N. bangense JCM 10635 & СР031305-СР031309 & $1.8 \pm 0.1$ & NA & $1.8 \pm 0.1$ \\
\hline H. limi JCM 16811 & СР031311-СР031314 & $3.0 \pm 0.3$ & a & $3.0 \pm 0.3$ \\
\hline H. salinum JCM 19729 & СР031310 & $1.8 \pm 0.1$ & NA & $1.8 \pm 0.1$ \\
\hline M. boonei 6A8 & СР000780 & $1.4 \pm 0.5$ & $2.1 \pm 0.7$ & $3.4 \pm 1.2$ \\
\hline
\end{tabular}

Values represent the mean \pm SD of three analyses of $10 \mu \mathrm{g}$ of archaeal DNA. Source data are provided as a Source Data file

NA, not applicable

andicates that the PT-linked dinucleotides were detected but were below the limit of quantification

per $10^{4} \mathrm{nt}$, which is comparable to the frequency of $\sim 3-8$ PT per $10^{4} \mathrm{nt}$ detected in bacteria ${ }^{16}$, prompting us to examine the PT features across the archaeal genomes (Table 1).

To this end, we exploited the single-molecule real-time (SMRT) sequencing platform to profile the genome-wide PT $\operatorname{sites}^{32,33}$. The SMRT sequencing data immediately revealed the occurrence of $d\left(\mathrm{G}_{\mathrm{PS}} \mathrm{A}\right)$ in a 4-bp consensus sequence of 5'$\mathrm{G}_{\mathrm{PS}} \mathrm{ATC}-3^{\prime}$ in all four haloarchaea genomes (Supplementary Fig. 2). Only $2.6 \%$ (2451) out of 94,316, 2.1\% (1614) out of $75,884,2.4 \%$ (1442) out of 60,776 , and $1.4 \%$ (895) out of 64,824 5'-GATC-3' sites in $H$. jeotgali A29, $N$. bangense JCM 10635, $H$. limi JCM 16811 and H. salinum JCM 19729 were detected to be PT modified, respectively (Supplementary Data 2). Although no further sequence constraint beyond the 4-bp 5'-G PSS ATC- $3^{\prime}$ was identified, we observed sequence biases for PT modificationproximate bases, with a noticeable preference for $5^{\prime}-\mathrm{tG}_{\mathrm{PS}} \mathrm{ATCc}-3^{\prime}$ but an underrepresentation at $5^{\prime}-\mathrm{gG} \mathrm{PS}_{\mathrm{PS}} \mathrm{ATC}(\mathrm{g} / \mathrm{t})-3^{\prime}$ (Supplementary Table 1). No PT enrichment in particular DNA regions was observed across all four archaeal genomes (Fig. 2 and Supplementary Data 2). For instance, 1229 and $12225^{\prime}-\mathrm{G}_{\mathrm{PS}} \mathrm{ATC}-3^{\prime}$ sites were observed to be located on the $(+)$ and $(-)$ strands of the $H$. jeotgali A29 genome, respectively, with various spacing ranging from 4 to $36 \mathrm{~kb}$. In addition, 915 out of 4745 open reading frames, 8 out of 13 rRNA genes and 31 out of 200 pseudogenes in $H$. jeotgali A29 harbour at least one PT site (Fig. 2 and Supplementary Data 2). While 1310 of 2451, 674 of 1614, 736 of 1442 , and 244 of 895 genomic PTs were detected to be fully PT-modified (on both strands) $5^{\prime}-\mathrm{G}_{\mathrm{PS}}$ ATC-3'/5'-G GS ATC-3' in $H$. jeotgali A29, $N$. bangense JCM 10635, H. limi JCM 16811 and $H$. salinum JCM 19729, respectively, up to $47 \%$ (1141), 58\% (940), $49 \%$ (706), and $73 \%$ (651) of PT sites occur in the form of

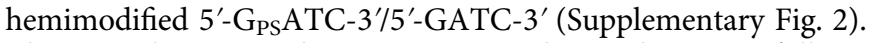
These results are in sharp contrast to the predominant full PT modification found in $5^{\prime}-\mathrm{G}_{\mathrm{PS}} \mathrm{AAC}-3^{\prime} / 5^{\prime}-\mathrm{G}_{\mathrm{PS}} \mathrm{TTC}-3$ in $E$. coli $\mathrm{B}^{3} \mathrm{~A}^{32}$ and $5^{\prime}-\mathrm{G}_{\mathrm{PS}} \mathrm{GCC}-3^{\prime} / 5^{\prime}-\mathrm{G}_{\mathrm{PS}} \mathrm{GCC}-3^{\prime}$ in Pseudomonas fluorescens pf0-134, suggesting different PT physiologies among Archaea. Additionally, it was interesting to observe the presence of DNA methylation in a variety of sequence contexts, including 5'-TCCGA ${ }^{6 \mathrm{~m}} \mathrm{~A}-3^{\prime}, \quad$ 5'-GAGG ${ }^{6 \mathrm{~m}} \mathrm{AG}-3^{\prime}, \quad 5^{\prime}-\mathrm{C}^{6 \mathrm{~m}} \mathrm{ATTC}-3^{\prime}, \quad 5^{\prime}-$ CAG ${ }^{6 \mathrm{~m}} \mathrm{ATG}-3^{\prime}$, and $5^{\prime}-\mathrm{CG}^{6 \mathrm{~m}} \mathrm{ATCC}-3^{\prime}$, by SMRT sequencing (Supplementary Table 2), which indicated their potential involvement in methylation-based R-M defence systems, epigenetic regulation, or other biological functions in Archaea.

DndCDEA constitutes a novel antiviral system with PbeABCD. Based on these PT characteristics in Archaea, we sought to explore the genomic neighbourhoods of $d n d C D$ by selecting 2361 out of 2642 archaeal and bacterial $d n d C D$ with available flanking sequence data. The restriction component $d n d F G H$ was observed to be in close proximity ( $1 \mathrm{bp}-20 \mathrm{~kb}$ ) with $48.8 \%$ (1132) of the
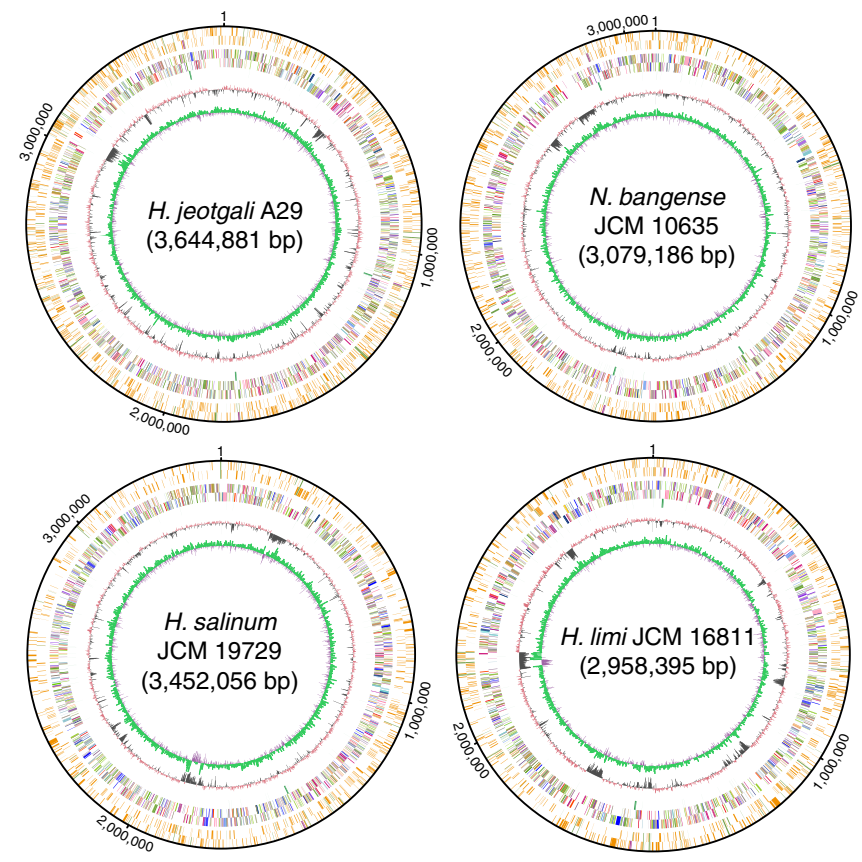

Fig. 2 Phosphorothioate (PT) mapping across the genomes of $H$. jeotgali A29, N. bangense JCM 10635, H. salinum JCM 19729 and H. limi JCM 16811. From the outer to the inner circles: circles 1 and 2 (forward and reverse strands), PT sites in ORFs (orange), noncoding RNA (purple), and nonencoding regions (green); circles 3 and 4, predicted protein-coding sequences coloured according to COG functional categories; circle 5, tRNA/rRNA operons; circle 6, guanine-cytosine content; and circle 7, guanine-cytosine skew. Source data are provided as a Source Data file

2322 bacterial $d n d C D$ regions, agreeing well with our previous observations ${ }^{34}$. Conversely, no trace of $d n d F G H$ was observed within the vicinity of all 39 archaeal $d n d C D$. Instead, the majority of these archaeal $d n d C D$ clusters are followed by a four-gene cassette arranged in a highly conserved order (Fig. 3).

Using the gene cassette consisting of DVR14_03960, DVR14_03955, DVR14_03950 and DVR14_03945 in H. jeotgali A29 as an example, the four genes are co-expressed as a single transcriptional unit, consistent with the idea that they are functional related (Supplementary Fig. 3). DVR14_03960 comprises three domains: a phospholipase D (PLD)-like domain (pfam13091), a res subunit of Type III REase (pfam04851) and a DEAD (Asp-Glu-Ala-Asp)-family helicase C-terminal (pfam00271) domain (Supplementary Fig. 4). The PLD domain has been identified as a component of a large superfamily of proteins associated with the hydrolysis of phosphodiester bonds, such as phospholipases, kinases, toxins and REases ${ }^{35}$. The genes 
a

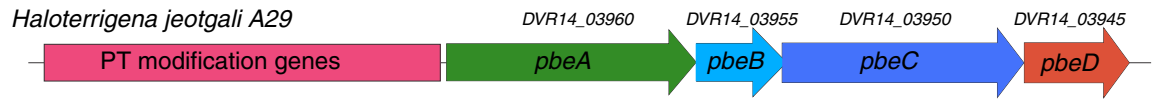

Salmonella enterica serovar Cerro 87
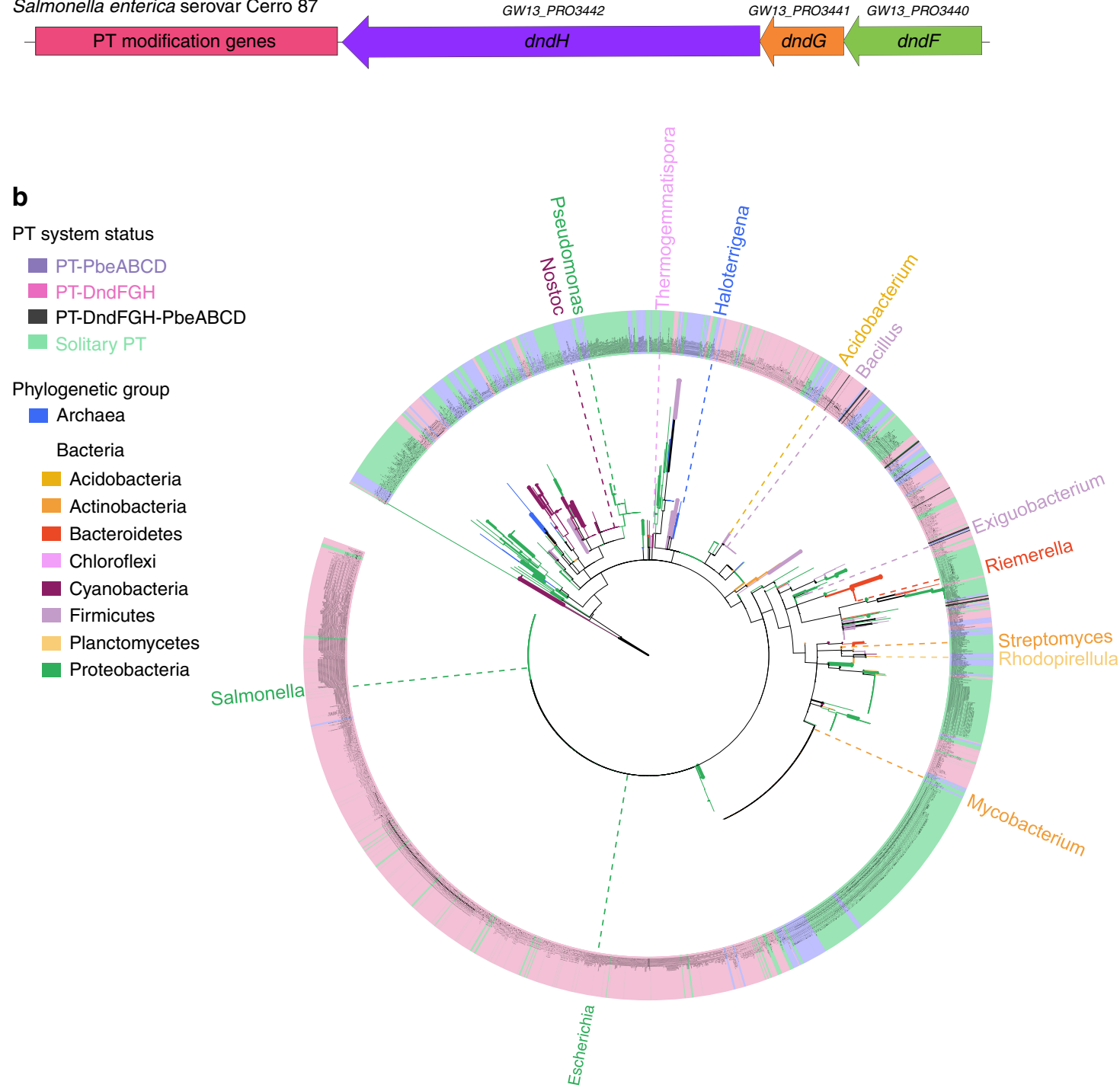

Fig. 3 The diversity of phosphorothioate (PT) systems in prokaryotes. a The genetic organisation and gene orders of the PT-based PbeABCD and DndFGH systems are displayed. GenBank accession numbers for pbeABCD in H. jeotgali A29 are indicated. b The phylogenetic tree of 2361 archaeal and bacterial DndCD homologues is colour-coded by phylogenetic group (see the legend). Strains shaded violet, pink, black and green have DndCD homologues associated with PbeABCD, DndFGH, DndFGH-PbeABCD, or none, respectively. The widths of tree branches are proportional to their Bootstrap scores. For clarity, at least one familiar genus per colour segment were labelled. Source data are provided as a Source Data file

DVR14_03955 and DVR14_03945 encode proteins of unknown function with only 66 and 152 aa in size, respectively. DVR14_03950 contains an N-terminal AAA domain (ATPases associated with diverse cellular activities; pfam13476), a domain that is present in a wide variety of proteins that often direct molecular remodelling events in an ATP-driven process ${ }^{36}$. Given the positioning of these genes relative to $d n d C D E A$ and the predicted nuclease-related functions of their encoded proteins, we speculated that this four-gene cluster may constitute a new defence system involving DNA PT modification in Archaea, and we named the four genes pbeABCD (for phosphorothioateblocked DNA exclusion).

To explore the involvement of pbeABCD in archaeal host-virus interactions, we cloned the $d n d C D E A-p b e A B C D$ fragment from
H. jeotgali A29 into the shuttle vector pFJ6-H, generating pWHU3808, followed by transformation into Natrinema sp. CJ7F, a pyrF-deletion and plasmid-free derivative of Natrinema sp. J7-1 (Supplementary Table 3). We next challenged CJ7-F cells expressing dndCDEA-pbeABCD with the halophilic temperate sphaerolipovirus SNJ1. Using a double agar overlay plaque assay, we showed that dndCDEA-pbeABCD provided protection against SNJ1: the ability of SNJ1 to induce plaques was reduced $\sim 10^{4}$-fold (Fig. 4).

To further examine the components that are essential for this antiviral activity, we constructed a series of pWHU3808-derived plasmids (pWHU3813 pWHU3816), each expressing DNA PT modification, as well as the individual in-frame deleted genes of the pbeABCD cassette (Supplementary Table 3). Deletion of the individual pbeABCD genes impaired protection against SNJ1, 


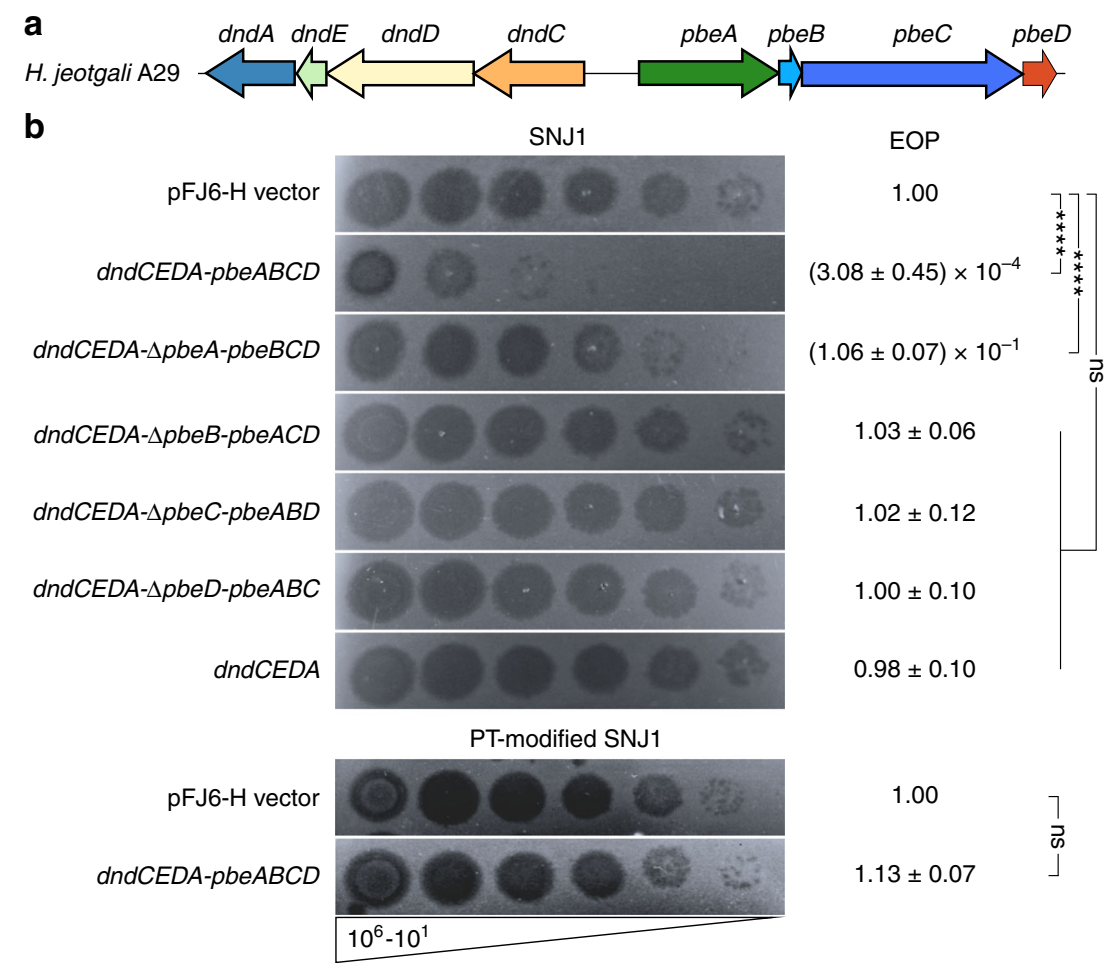

Fig. 4 DndCDEA-PbeABCD confers protection against the haloarchaeal virus SNJ1. a The dndCDEA-pbeABCD locus of $H$. jeotgali A29 is displayed. b Five microlitres each of a tenfold serial dilution $\left(10^{6}-10^{1}\right)$ of SNJ1 virus was spotted onto CJ7-F cells carrying pWHU3808-derived plasmids and expressing the genes indicated on the left. The EOPs are listed on the right. The EOPs were determined by dividing the SNJ1 titre obtained by plating on CJ7-F expressing dndCDEA-pbeABCD and derivatives by the titre of SNJ1 plated on CJ7-F(pFJ6-H). The values represent the mean \pm SD for three independent experiments. ${ }^{\star \star \star *} p$-value $<0.0001$, using one-sample $t$-test (Hypothesis Testing). Ns, not significant. Compared to the strong protection against non-PT SNJ1, CJ7-F cells are susceptible to PT-modified SNJ1, even in the presence of DndCDEA-PbeABCD. Source data are provided as a Source Data file

indicating that all four pbe genes are indispensable for mediating antiviral activity (Fig. 4). It is noteworthy that the contributions of four pbe genes against SNJ1 are not equal. To quantify the protection, we measured the efficiency of plating (EOP) of SNJ1 applied to CJ7-F cells expressing different parts of the pbeABCD cassette. In contrast to the absolute necessity of $p b e B, p b e C$ and pbeD, CJ7-F cells expressing dndCDEA- $\triangle p b e A-p b e B C D$ still exhibited a low-level of resistance, although less than one order of magnitude, against $\mathrm{SNJ} 1$, suggesting that pbeA is partially required for defence (Fig. 4). To assess whether PT modification is sufficient to protect viruses against $\mathrm{PbeABCD}$ interference, we propagated SNJ1 in CJ7-F cells expressing only dndCDEA, yielding PT-modified SNJ1 (Supplementary Fig. 5). In sharp contrast to the strong protection observed against the non-PTmodified SNJ1 virus, CJ7-F cells expressing dndCDEA-pbeABCD displayed the same susceptibility to PT-modified SNJ1 as CJ7-F cells lacking dndCDEA-pbeABCD (Fig. 4).

It is, therefore, likely that $d n d C D E A-p b e A B C D$ encodes $\mathrm{R}-\mathrm{M}$ activity for which DNA PT modification serves as a recognition tag, allowing discrimination between self and non-self DNA. If the Pbe moiety is capable of independent action, then deletion of PT modification genes would likely be detrimental to archaeal cells because genomic 5'-GATC-3' sites would no longer be protected by PT from the restriction activity of PbeABCD. Surprisingly, it was easy to prepare the plasmids pWHU3809, pWHU3810, pWHU3811 and pWHU3812 harbouring individually in-frame-deleted $d n d C D E A$ genes but a complete pbeABCD (Supplementary Table 3). Indeed, these plasmids exerted no notable toxicity towards CJ7-F cells and failed to reduce the ability of SNJ1 virus to cause plaque formation, despite the expression of pbeABCD (Supplementary Fig. 6).
Thus, it is possible that the antiviral activity of PbeABCD depends on DNA PT modification in trans or on DndCEDA proteins. To address this possibility, we attempted to construct a plasmid (pWHU3789) expressing DndCDEA C344S $_{\text {-PbeABCD }}$ with a single cysteine replaced by serine in DndA. The catalytic cysteine of DndA (C344), corresponding to C327 in S. lividans DndA, is responsible for the nucleophilic attack on the substrate cysteine $^{37}$. Thus, although this mutation would abolish DNA PT modification at $5^{\prime}-\mathrm{G}_{\mathrm{PS}} \mathrm{ATC}-3^{\prime}$, the integrity of the DndCDEA assembly would be retained. The immediate observation was that in spite of the deficiency of PT modification, pbeABCD was still transcribed in CJ7-F(dndCDEA $A_{C 344 S}$, pbeABCD), excluding the possibility that DndA or PT acts as a transcriptional regulator of the pbe operon (Supplementary Fig. 7a, b). However, while the transformation of CJ7-F hosts with the pWHU3789 plasmid occurred at normal frequency, the CJ7-F(pWHU3879) transformant restored the sensitivity to SNJ1 (Supplementary Fig. 7c). This result suggested that PbeABCD exerted antiviral activity depending on the presence of PT modifications rather than protein-protein interactions with DndCDEA. This activity differentiates the PbeABCD-mediated defence mechanism from that of DndFGH because the latter still exerted a bactericidal effect, even in the absence of PT modification.

PbeABCD allows virus adsorption but inhibits DNA replication. To gain further insight into the mechanism of action of the PbeABCD-mediated PT defence system, we examined whether the system provides protection by hindering virus attachment. To this end, we compared the efficiency of SNJ1 adsorption to dndCDEApbeABCD-containing and dndCDEA-pbeABCD-lacking CJ7-F 
cells. The result showed that SNJ1 viruses were able to adsorb to the archaeal cells at the same rates regardless of the presence of the dndCDEA-pbeABCD system (Fig. 5a). This finding suggests that DndCDEA-PbeABCD does not offer protection by interfering with virus attachment.

We next monitored the replication efficiency of SNJ1 viral DNA during the time course of the infection using real-time quantitative polymerase chain reaction (qPCR). The PCR products for $r e p A$, which is located within the replication region of SNJ1, and $\mathrm{radA}$, a single-copy gene located on the CJ7-F chromosome, were used to estimate the relative viral DNA concentrations compared to archaeal genome equivalents ${ }^{38}$. After $5 \mathrm{~h}$ of infection, a 2.5 -fold increase in the intracellular SNJ1 genome level was observed in CJ7-F (dndCDEA, pbeABCD), whereas a 16.7-fold increase was detected in CJ7-F containing the empty vector (Fig. 5b). These results revealed that SNJ1 DNA can be successfully injected into Natrinema cells but that the subsequent viral genome replication process is significantly inhibited by the DndCDEA-PbeABCD system. This observation, along with the results of the adsorption assays, indicated that the replication inhibition likely results from viral DNA cleavage or degradation.

To examine the integrity of SNJ1 DNA, we performed a Southern blot analysis using total DNA isolated from CJ7-F cells treated with SNJ1 at various time points after infection. With increasing infection time, extensive SNJ1 DNA accumulation was observed in dndCDEA-pbeABCD-lacking but not $d n d C D E A$ pbeABCD-containing CJ7-F cells, indicating that DndCDEAPbeABCD halts viral infection at an early stage (Fig. 5c). Notably, SNJ1 DNA remained intact with no sign of cleavage or processive degradation, even $6 \mathrm{~h}$ after infection of CJ7-F cells expressing the DndCDEA-PbeABCD system (Fig. 5c). Consistent with the real-
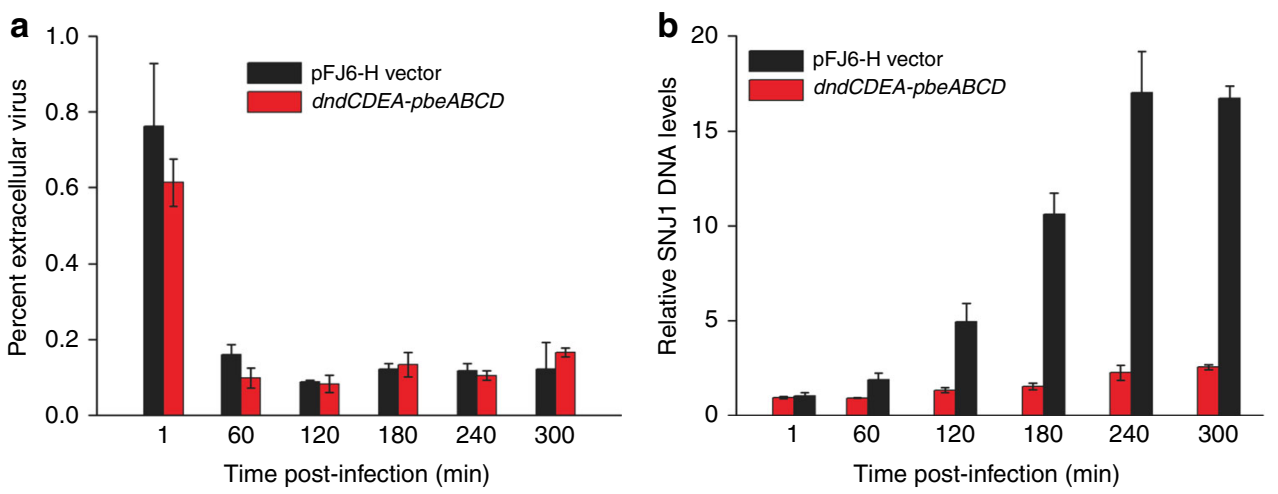

C dnd $C D E A-p b e A B C D$

pFJ6-H vector

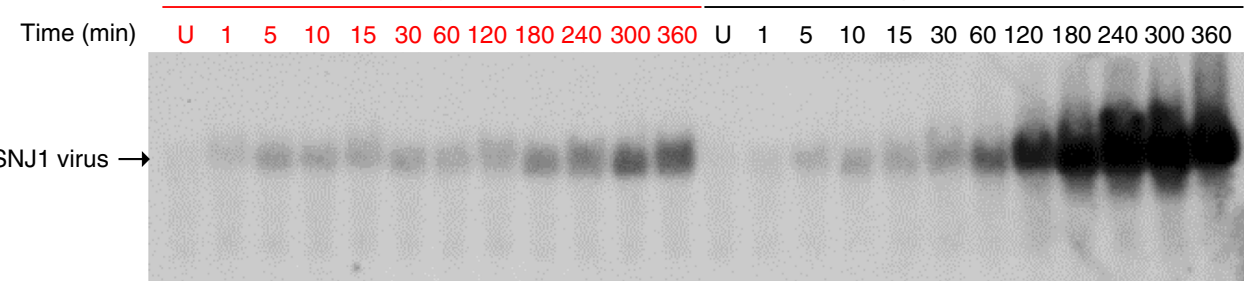

Fig. 5 Adsorption and DNA replication of the SNJ1 virus in CJ7-F cells expressing or lacking DndCDEA-PbeABCD. a Adsorption of SNJ1 to dndCDEApbeABCD-containing CJ7-F cells (red) is not impaired compared to that observed for CJ7-F cells containing the empty vector pFJ6-H (black). After the infection of logarithmic-stage cultures (OD600 $=0.3$ ) with SNJ1 at an $\mathrm{MOI}=1$, samples were collected at 60 -min intervals, and the extracellular (unabsorbed) viral concentration was measured and compared to the initial viral concentrations. The bars represent the means of three experiments, and the error bars represent the SD of the mean. $\mathbf{b}$ Real-time qPCR analysis to determine the replication efficiency of the virus SNJ1 in CJ7-F cells expressing (red) or lacking (black) dndCDEA-pbeABCD. Primers amplifying repA and radA were used to determine the concentration of SNJ1 DNA and CJ7-F chromosomes, respectively. The $2 \mathrm{C}_{T}(\mathrm{radA})-\mathrm{C}_{\mathrm{T}}$ (repA) method was used to determine the relative levels of SNJ1 DNA in CJ7-F hosts. c Southern blot analysis of the SNJ1 genome during the infection cycle. Numbers indicate the time ( $\mathrm{min}$ ) following infection. A probe was designed corresponding to positions 9243-9732 nt in the SNJ1 genome. Source data are provided as a Source Data file 
Considering the functional association, we next examined whether these PT modification components had coevolved and been horizontally transferred together with PbeABCD, similar to what occurred with DndFGH ${ }^{34}$. Using DndCD and PbeAC of $H$. jeotgali A29 as references, we calculated the alignment similarity rate between the $261 \mathrm{DndCD}$-PbeAC pairs and their respective reference Dnd and Pbe proteins using the approach previously described $^{34}$. The same calculation was applied to the 1132 coexisting DndCD-DndFGH proteins, with DndFGH of $S$. enterica serovar Cerro 87 used as the reference. In general, the coevolution of the modification and restriction components should lead to a linear relationship between their similarity rates $^{34}$. The results showed that the correlation coefficient $(\rho)$ between the similarity rates for DndCD and DndFGH was 0.582 , which is consistent with our previous report ${ }^{34}$ and confirms the coevolution of DndCD and DndFGH (Supplementary Fig. 8a). In contrast, a $\rho$-value of 0.107 between DndCD and PbeAC was observed, suggesting that either the $d n d C D$ and pbeAC genes were acquired separately by HGT events or that they evolved at different mutational rates owing to different genetic selection pressures (Supplementary Fig. 8b).

To gain a more global view of this new type of PT-based PbeABCD defence system, we performed homology searches and constructed a phylogenetic tree using all 553 PbeAC proteins detected (Fig. 6). An immediate observation was the occurrence of potential interdomain and intradomain gene transfer events. For instance, instead of clustering with other halophilic Archaea, Halorubrum trapanicum CBA1232 grouped with a large clade of bacterial strains, including those of the genera Lactobacillus, Staphylococcus and Bacillus. Surprisingly, only 42.1\% (233) of 553
PbeAC proteins were observed to be accompanied by $d n d C D$ within $1 \mathrm{bp}-10 \mathrm{~kb}$, raising two possibilities: (1) that the solitary PbeABCD components are not active in host cells because their antiviral activity depends on the presence of PT modification and (2) that PbeABCD has evolved to pair with other DNA modifications, such as methylation, to yield new defensive modules. The latter possibility is supported by the observation that candidate base-modifying proteins are encoded within $10 \mathrm{~kb}$ of 155 of 320 solitary PbeAC examples (Supplementary Data 4). Notably, PbeAC and DndFGH were observed to be simultaneously present in the neighbourhood of 25 bacterial $d n d C D$ clusters (Fig. 3 and Supplementary Data 3). It would be interesting to explore the interactions between these gene cassettes and their contributions to the host defence against invasive DNA.

\section{Discussion}

In this study, we describe a new type of PT-based multi-gene antiviral system in Archaea, DndCDEA-PbeABCD, that is genetically and phenotypically distinct from DndBCDEDndFGH. In spite of the absence of the transcriptional repressor DndB, the DndCDEA-PbeABCD system in four haloarchaeal strains drives motif-specific DNA PT modifications at frequencies comparable to those observed in Bacteria ${ }^{32,34}$. Approximately half of PT sites occur in the form of hemi-PT-modified 5'-G - $_{\mathrm{PS}} \mathrm{ATC}-3^{\prime} /$ 5'-GATC-3' in Archaea, which is in sharp contrast to the predominant full PT modifications that occur in Bacteria ${ }^{32,34}$. However, although PbeABCD allows viral adsorption and DNA injection into the host archaeal cells, it prevents viral DNA

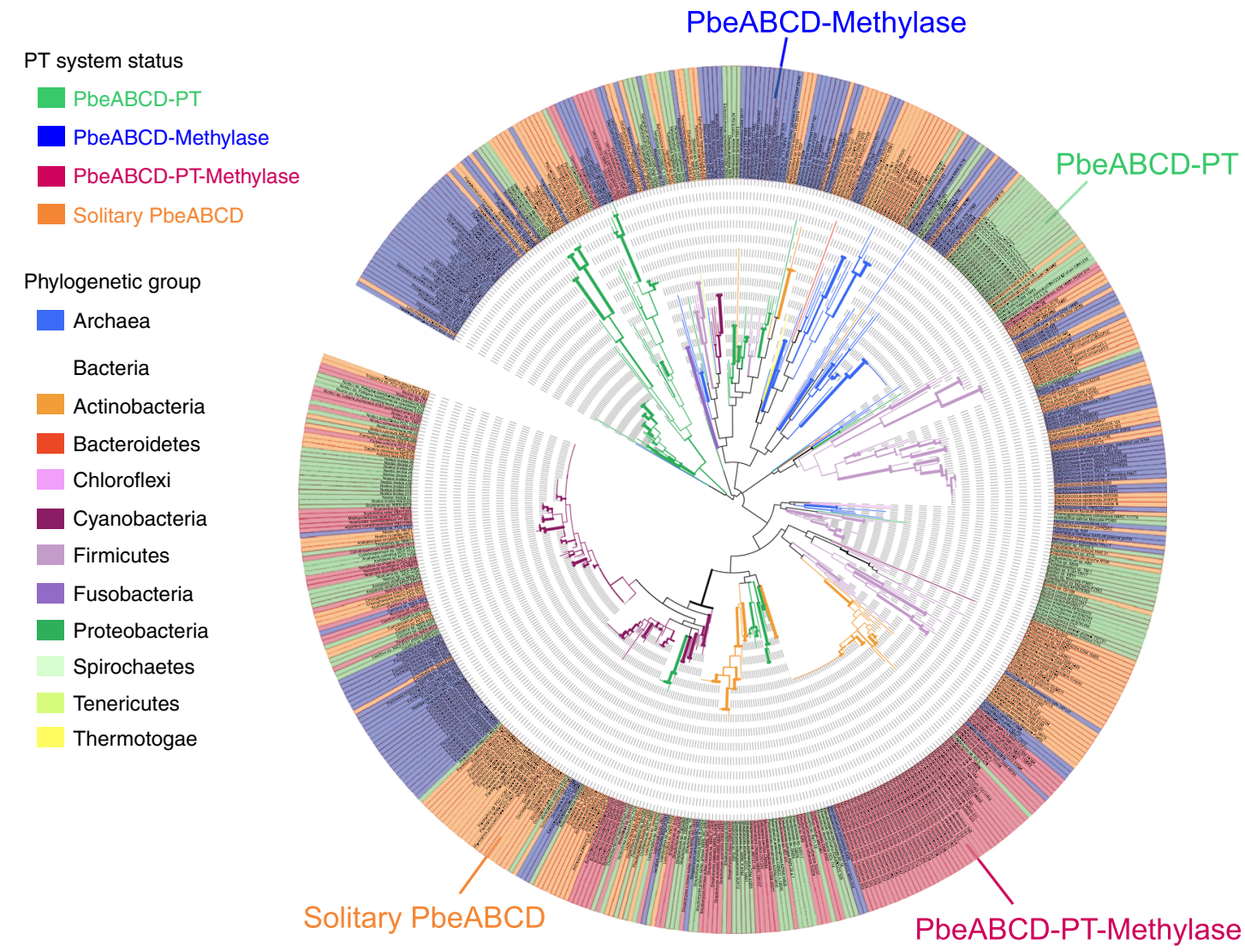

Fig. 6 Phylogenetic analysis of 553 PbeAC proteins. Each archaeal and bacterial group is represented in a distinct colour. Green (128) and blue (155) shading indicate the presence of DndCD for phosphorothioate (PT) modification or MTase for methylation within the vicinity of PbeAC. One-hundred and five PbeAC homologues have DndCD and MTase simultaneously present within the defined neighbourhood (1 bp-10 kb), while 165 PbeAC homologues are solitary, lacking the modification component within the neighbourhood. Source data are provided as a Source Data file 
replication in a PT-dependent manner. Notably, the DndCDEAPbeABCD pair was no longer able to protect against a PTmodified SNJ1 virus, suggesting a strategy of utilising epigenetic modification for self/non-self discrimination. We therefore speculated that $\mathrm{PbeABCD}$ serves as the REase component to target non-PT-modified viral DNA, analogous to R-M systems. However, two lines of evidence point to a novel mechanism of defence that is different from type II R-M systems, which have autonomous REase and MTase activities, and PT-based DndFGH systems. First, no sign of the processive degradation or cleavage of viral DNA was detected in the presence of DndCDEA-PbeABCD. Second, in typical R-M or DndFGH-mediated Dnd systems, the loss of methylation or PT would be detrimental to cells because the resulting host DNA would be recognised as invading foreign DNA and be attacked by REases or DndFGH. However, dndCDEA deletion mutants exhibited no apparent growth defect compared to CJ7-F cells expressing intact dndCDEA-pbeABCD, although protection against the virus was lost.

We also showed that all four genes in the pbe $A B C D$ module appear to be essential for protection against viral infection. PbeA consists of a PLD domain ${ }^{35}$, a Res subunit of Type III REase domain and a helicase domain ${ }^{39,40}$, suggesting that it has multiple enzymatic activities. The PLD domain is present in a wide variety of enzymes involved in phospholipid metabolism, virulence, membrane remodelling and signalling ${ }^{35}$. In addition, it is also recognised as a characteristic of a small family of endonucleases in bacteria, such as Nuc, CglI, NgoAvII, BfiI and DrmC, a component of the newly identified multi-gene R-M module DISARM ${ }^{2,41-43}$. The Res subunit of Type III REase (i.e., EcoP15I) is a fusion between a DEAD-family helicase, correlating with the DNA translocation activity, and a nuclease resembling the functionally related HsdR subunit of the type I REases ${ }^{39,40}$. Nonetheless, the PD-X-D/EXK motif, the nuclease active site for DNA cleavage, is not present in $\mathrm{PbeA}$, which is consistent with the nonDNA-cleavage defensive activity of PbeABCD. Based on the shared helicase motifs between PbeA and type I and III REases, we, speculate that PbeA might be associated with DNA translocation, looping or sliding to target DNA recognition sites. This possibility would explain why without $p b e A$, the remaining $p b e B C D$ still confer a low-level of resistance to SNJ1. While $p b e B$ and $p b e D$ encode proteins of unknown function sized only 66 and 152 aa, respectively, deletions of them still completely abolished protection against virus SNJ1. Considering their small size, we speculate that $\mathrm{PbeB}$ and $\mathrm{PbeD}$ might become part of an enzyme complex for stabilisation or functional coupling. Notably, PbeC exhibits sequence resemblance to the structural maintenance of chromosome (SMC) protein ( $24 \%$ identity and $42 \%$ similarity) in Hydrococcus rivularis. SMC proteins adopt a V-shaped, twoarmed architecture with an ATP-binding cassette (ABC)-like domain at the distal end of each arm and are recognised as one of the most fundamental classes of proteins involving in chromosome segregation, recombinational repair and genome-wide gene regulation (Supplementary Data 5) ${ }^{44}$. Although the function and interaction of $\mathrm{PbeABCD}$ proteins remain to be further elucidated, these preliminary clues indicate a possible mechanism of action whereby viral DNA molecules are manipulated in a certain manner to inhibit viral replication and propagation. In terms of the PT dependence, we speculate that Pbe protein(s) might sense PT DNA to function or that PbeABCD rely on PT to form an active assembly.

It was interesting to observe the potential HGT events of DndCD between different bacterial phyla, as well as between Archaea and Bacteria. However, based on the phenomenon that DndFGH occurs only in Bacteria and not in Archaea, this interdomain HGT situation does not hold for the restriction component DndFGH. Instead, the PT modifications in Archaea have evolved to be paired with PbeABCD, generating a new type of PT-based defence module with mechanisms of action that differ from those of DndFGH. In addition, PbeABCD-mediated PT defence pairs are also widespread among Bacteria. Further phylogenetic analysis of PbeAC revealed two unusual observations: (1) 320 of 553 pbeAC are solitary, lacking the counterpart PT modification dnd genes; and (2) pbeAC, dndFGH and PT modification genes coexist in 25 bacterial strains. Regarding the occurrence of solitary pbeAC, it is possible that they are not functional due to their dependence on PT. This scenario is different from that of the REases in type I and III R-M systems, which are activated via protein-protein interactions with the cognate MTases to enable their restriction activity. At present, we still cannot rule out the possibility that PbeABCD has evolved to pair with other modification counterparts, such as MTases, to generate new defensive modules, which is supported by the observation that 155 of 320 solitary pbeAC components are in close proximity to genes encoding MTases. In terms of the coexistence of DndFGH, PbeABCD and PT modifications, it is currently unclear whether the first two are both functional or which one pairs with PT or methylation as a defensive module, emphasising the possibility of complicated interactions worthy of future investigation.

In the host-parasite coevolutionary arms race, viruses have developed a variety of counter-resistance tactics to circumvent the arsenal of prokaryotic resistance mechanisms ${ }^{5}$. Considering that the PT-modified SNJ1 virus can bypass PbeABCD-mediated PT defence systems, we can speculate that once the virus completes an infection cycle, the resulting SNJ1 progeny possessing PT modifications would overcome this defence in other archaeal and bacterial cells, regardless of the presence of PbeABCD or DndFGH. Thus, in future studies, it would be interesting to identify viruses with naturally occurring PT modifications or to explore other strategies used by viruses to avoid, circumvent or subvert PT-based defence systems.

Owing to the fairly limited number of archaeal host-virus systems studied, our understanding of archaeal host-virus interactions lags far behind our understanding of those in Bacteria. These limitations also hinder the exploration of new defensive mechanisms in Archaea. In this study, we identified a new type of PT-based Dnd defence system that is widespread in Archaea and Bacteria. The antiviral activity of PbeABCD depends on the presence of DNA PT modification but does not involve viral DNA degradation or cleavage, ruling out the involvement of currently known defence mechanisms. These findings expand our knowledge regarding the arsenal of archaeal defence systems and illuminate the archaeal host-virus arms race.

\section{Methods}

Strains and growth conditions. All the strains, plasmids and primers used in this study are listed in Supplementary Tables 3 and 4. Natrinema sp. CJ7-F and its derivative strains were cultured in Halo-2 or minimal medium (MM, 18\%) at $45^{\circ}$ C, as previously described ${ }^{45}$. H. jeotgali A29, H. limi JCM 16811 and H. salinum JCM 19729 were cultivated in JCM medium 402, and N. bangense JCM 10635 was grown in JCM medium 167. To construct plasmids, E. coli strains were routinely grown in Luria-Bertani (LB) medium at $37^{\circ} \mathrm{C}$. To prepare solid agar medium, agar was added to the medium to a final concentration of $15 \mathrm{~g} / \mathrm{L}$. When necessary, ampicillin was added to the medium at a concentration of $100 \mu \mathrm{g} / \mathrm{mL}$.

Construction and expression of pWHU3808 and derivatives. The shuttle vector pFJ6-H was used to express dndCDEA-pbeABCD from H. jeotgali A29 and its derivatives in $\mathrm{CJ} 7-\mathrm{F}^{45}$. Plasmid pWHU3808, expressing the complete dndCDEApbeABCD module, was constructed in two steps. First, the dndCDEA and pbeABCD fragments were PCR amplified from $H$. jeotgali A29 genomic DNA using the primer pairs dndCDEA-pYC-F/dndCDEA-pYC-R and pbeABCD-F/pbeABCD-R, respectively. The fragments were inserted into the SnaBI-HindIII-digested pYCJ$\mathrm{HH}$ and NotI-SphI-digested pFJ6-H, yielding pWHU3253 and pWHU3804 respectively. Second, the heat-shock protein 70 (HSP 70) promoter and the dndCDEA fusion fragments were PCR amplified from pWHU3253 using the 
primer pair Hpro-dndCDEA-F/Hpro-dndCEDA-R. The PCR product was then ligated into pWHU3804 digested with NdeI and KpnI, yielding pWHU3808. For the in-frame deletion of pbeA in pWHU3808, a $5.2 \mathrm{~kb}$ fragment containing the downstream portion of $p b e A$ was first generated by PCR using the primer pair $\Delta$ pbeA-F/ $\triangle$ pbeA-R from pWHU3808 (Supplementary Table 4 ). The PCR products and the NdeI-NotI digested pWHU3808, sharing $25-30$ bp identical sequences, were mixed to allow site-specific recombination using a Hieff Clone ${ }^{\text {mt }}$ Plus One Step Cloning Kit (Yeasen), generating plasmid pWHU3813. To generate the in-frame deletion of $p b e B$ in pWHU3808, the upstream and downstream fragments of pbeB were PCR amplified using the primer pairs $\Delta$ pbeB-UF/ $\Delta$ pbeB-UR and $\Delta$ pbeB-DF/ $\Delta$ pbeB-DR, respectively (Supplementary Table 4 ). The two purified PCR products and the NdeI-NotI-digested pWHU3808 were mixed according to the manufacturer's instructions for recombination to generate the plasmid pWHU3814. The procedures used to construct the other pWHU3808-derived plasmids expressing in-frame deletions of the $d n d$ genes were similar to those used for pWHU3814. All of the recombinant plasmids were verified by sequencing. The transformation of CJ7-F was performed at room temperature using the polyethylene glycol (PEG) method, as previously described ${ }^{38}$. MM (18\%) medium was used to select and propagate CJ7-F transformants.

\begin{abstract}
Absorption assay. CJ7-F cells expressing or lacking $d n d C D E A-p b e A B C D$ were grown in Halo-2 medium to an $\mathrm{OD}_{600}$ of 0.3 , followed by infection with virus SNJ1 at a multiplicity of infection (MOI) of 1. During infection, the archaeal cells and virus were incubated with shaking at $45^{\circ} \mathrm{C}$. Aliquots of $1 \mathrm{~mL}$ of culture were withdrawn at time points $1,60,120,180,240$ and $300 \mathrm{~min}$ post infection, and the cells were pelleted by centrifugation at $10,000 \times g$ for 1 min. The unabsorbed SNI1 concentration in the upper aqueous phase was determined on CJ7-F cell lawns. The percentage of unabsorbed (extracellular) virus SNJ1 was calculated assuming the initial titre of SNJ1 (without added cells) to be $100 \%$.
\end{abstract}

Determination of relative SNJ1 levels in CJ7-F hosts. CJ7-F cells harbouring pWHU3808 or the empty vector pFJ6-H, expressing or lacking $d n d C D E A-$ pbe $A B C D$, respectively, were cultured in $20 \mathrm{~mL}$ of Halo- 2 medium until the cell density reached an OD600 of 0.3. Logarithmic-phase cultures were infected with the SNJ1 virus at an MOI of 1. Multiple 1-mL samples of the cultures were collected at the indicated time point by centrifugation, and the cells were resuspended in $100 \mu \mathrm{L}$ of basal salt solution (medium without a carbon source). Total DNA from CJ7-F strains containing the different vectors was prepared as described previously ${ }^{46}$. Briefly, cells resuspended in $100 \mu \mathrm{L}$ of basal salt solution were lysed after the addition of $900 \mu \mathrm{L}$ of distilled water. The lysate was diluted 1:100 with distilled water and then was used as the template for real-time qPCR. The levels of the single-copy gene $\mathrm{radA}^{38}$, located on the chromosome of the host strain, and repA, located in the genome of SNJ1, were determined by real-time qPCR to estimate the relative intracellular levels of SNJ1 DNA relative to the CJ7-F chromosome during infection.

RNA extraction and reverse transcription. Total RNA was extracted from the $H$. jeotgali A29 strain using an Omega RNA Extraction Kit (Omega Bio-Tec) according to the manufacturer's protocol. Next, the RNA samples were treated with DNase I (Thermo Fisher) to remove residual genomic DNA, and the RNA was then quantified using a NanoDrop2000 spectrophotometer (Thermo Fisher). The RNA was reverse transcribed using a RevertAid First Strand cDNA Synthesis Kit (Fermentas) to obtain cDNA.

SNJ1 propagation and isolation. The haloarchaeal virus SNJ1 was propagated in Natrinema sp. J7- $1^{45}$. The J7-1 strain was grown at $45^{\circ} \mathrm{C}$ in Halo-2 medium to late log phase $\left(\mathrm{OD}_{600}=0.8-1.0\right)$, after which mitomycin $\mathrm{C}$ (Roche) was added to the cultures at a final concentration of $1 \mu \mathrm{g} / \mathrm{mL}$, followed by overnight incubation at 37 ${ }^{\circ} \mathrm{C}$ with shaking at $200 \mathrm{rpm}$. The cultures were centrifuged at $10,000 \times g$ for $20 \mathrm{~min}$ to remove cellular debris, and the supernatants were subsequently filtered through $0.22-\mu \mathrm{m}$ Millipore pore membrane filters. When necessary, a PEG6000 solution was used to concentrate the phage preparations, as described previously ${ }^{47}$.

Plaque assays. Halovirus plaque assays were performed as described previously ${ }^{48}$, with slight modifications. Briefly, the CJ7-F derivative strains were grown at $45^{\circ} \mathrm{C}$ in $18 \% \mathrm{MM}$ medium to late log phase $\left(\mathrm{OD}_{600}=\sim 0.8-1.0\right)$. Next, an aliquot $(400$ $\mu \mathrm{L}$ ) of the culture was mixed with molten soft agar $(0.6 \%)$ and poured onto Halo-2 agar plates (1.5\%). Once solidified, $5 \mu \mathrm{L}$ of serial tenfold dilutions of SNJ1 were pipetted onto the agar. The plates were incubated for $36 \mathrm{~h}$ at $45^{\circ} \mathrm{C}$, and the phage titres were calculated as PFU (plaque-forming unit) $/ \mathrm{mL}$.

Southern blot analysis. CJ7-F cells possessing pWHU3808 and pFJ6-H were incubated with the SNJ1 virus at an MOI of 5. Multiple aliquots of $10 \mathrm{~mL}$ of culture were collected at different time points $(1,5,10,15,30 \mathrm{~min}$ and $1,2,3,4,5$ and $6 \mathrm{~h})$ post infection. In addition, samples of the cultures prior to the addition of SNJ1 were used as negative controls. Total genomic DNA was extracted and digested with SacI for Southern blot analysis. The primers SNJ1-southern-F and SNJ1southern- $\mathrm{R}$ were designed to synthesise a probe corresponding to the region from
9243 to $9732 \mathrm{nt}$ of the SNJ1 genome (Supplementary Table 4). The DNA probe preparation, hybridisation and detection were performed using a Detection Starter Kit II (Roche) according to the manufacturer's instructions.

Bioinformatic and statistical analysis. Using the DndC, DndD, PbeA and PbeC protein sequences from $H$. jeotgali A29 and the DndF, DndG, and DndH sequences from S. enterica serovar Cerro 87 and Hahella chejuensis KCTC 2396 as BLAST queries, we searched the NCBI databases of non-redundant nucleotide collections, RefSeq_genomes, WGS, HTGS, refseq_genomic, and GSS. The BLAST hits were filtered using an $e$-value of $\leq 10^{-10}$ and an aligned length of $\geq 30 \%$ to the query sequences. The distance between $d n d C$ and $d n d D$ was limited to $5 \mathrm{~kb}$ or less. Flanking regions of $\pm 20 \mathrm{~kb}$ around the $d n d C D$ cluster were searched for the presence of pbeA, pbeC, $d n d F, d n d G$ and $d n d H$. Similarly, we searched for genes encoding MTases or DndCD within close proximity ( $1 \mathrm{bp}-10 \mathrm{~kb}$ ) to pbeAC.

The sequences used to generate the phylogenetic trees were formed by concatenating the protein sequences of DndC and DndD, and PbeA and PbeC. Multiple alignments were performed using MEGA 5.2 with the MUSCLE algorithm. The parameters were set as follows: gap open $=-2.9$, gap extend $=0$,

hydrophobicity multiplier $=1.2$, $\max$ iterations $=8$, and min dialogue length $=24$, and the UPGMB method was used for clustering. Trees were constructed using the maximum likelihood method with 500 bootstrap replications. The other parameters were set as follows: substitution type $=$ amino acid, substitution model $=$ JonesTaylor-Thornton model, rates among sites $=$ uniform rates, gaps $/$ missing data treatment $=$ complete deletion, maximum likelihood heuristic method $=$ NearestNeighbour-Interchange, and the neighbour-joining method was used to constructed the initial tree. The output trees were visualised by iTOL $^{49}$.

DndCD and PbeAC sequences wereglobally aligned with those of $H$. jeotgali A29 using the needle programme in the EMBOSS package ${ }^{50}$. Similarity values were extracted from the output files. The coevolution analysis has been described in detail previously ${ }^{34}$. Generally, we regressed the similarity values of PbeAC to those of DndCD to explore their coevolution on the basis of the idea that the divergence degree (i.e., 1 minus the similarity rate) of PbeAC and DndCD from their respective ancestor should be linearly related in a given strain, if they evolve at the same speed. The same regression analysis was also performed on DndCD and DndFGH. As $H$. jeotgali A29 lacks $d n d F G H$, global alignments were made with the sequence data from S. enterica serovar Cerro 87.

All statistical and bioinformatic analyses were performed in R.

\section{Data availability}

The H. jeotgali A29, N. bangense JCM 10635, H. salinum JCM 19729 and H. limi JCM 16811 sequencing data generated using the PacBio RSII platform have been deposited in the Sequence Read Archive under the accession numbers SRR7957355, SRR7957354, SRR7957356 and SRR7945240, respectively. The source data for Table 1, Figs. 1 to 6, Supplementary Tables 1 and 2, and Supplementary Figs. 1, 2, 3, 5, 6, and 7 are provided as a Source Data file. The data that support the findings of this study are available from the authors upon request.

\section{Code availability}

Custom codes or software used in this study is available from the authors upon request.

Received: 6 November 2018 Accepted: 7 March 2019

Published online: 11 April 2019

\section{References}

1. Swarts, D. C. et al. DNA-guided DNA interference by a prokaryotic Argonaute. Nature 507, 258-261 (2014).

2. Ofir, G. et al. DISARM is a widespread bacterial defence system with broad anti-phage activities. Nat. Microbiol 3, 90-98 (2018).

3. Goldfarb, T. et al. BREX is a novel phage resistance system widespread in microbial genomes. EMBO J. 34, 169-183 (2015).

4. Xu, T., Yao, F., Zhou, X., Deng, Z. \& You, D. A novel host-specific restriction system associated with DNA backbone S-modification in Salmonella. Nucleic Acids Res. 38, 7133-7141 (2010)

5. Labrie, S. J., Samson, J. E. \& Moineau, S. Bacteriophage resistance mechanisms. Nat. Rev. Microbiol. 8, 317-327 (2010).

6. Mao, S. Maps of defense arsenals in microbial genomes. Science 359 1004-1006 (2018).

7. Roberts, R. J. et al. A nomenclature for restriction enzymes, DNA methyltransferases, homing endonucleases and their genes. Nucl. Acids Res. 31, 1805-1812 (2003).

8. Murray, N. E. Type I restriction systems: Sophisticated molecular machines (a legacy of Bertani and Weigle). Microbiol. Mol. Biol. Rev. 64, 412-434 (2000).

9. Rao, D. N., Dryden, D. T. \& Bheemanaik, S. Type III restriction-modification enzymes: a historical perspective. Nucl. Acids Res. 42, 45-55 (2014). 
10. Sutherland, E., Coe, L. \& Raleigh, E. A. McrBC: a multisubunit GTPdependent restriction endonuclease. J. Mol. Biol. 225, 327-348 (1992).

11. Bair, C. L. \& Black, L. W. A type IV modification dependent restriction nuclease that targets glucosylated hydroxymethyl cytosine modified DNAs. J. Mol. Biol. 366, 768-778 (2007).

12. Xu, S. Y., Corvaglia, A. R., Chan, S. H., Zheng, Y. \& Linder, P. A type IV modification-dependent restriction enzyme SauUSI from Staphylococcus aureus subsp. aureus USA300. Nucl. Acids Res. 39, 5597-5610 (2011).

13. Wang, L. et al. Phosphorothioation of DNA in bacteria by dnd genes. Nat. Chem. Biol. 3, 709-710 (2007)

14. Gan, R. et al. DNA phosphorothioate modifications influence the global transcriptional response and protect DNA from double-stranded breaks. Sci. Rep. 4, 6642 (2014).

15. Eckstein, F. Phosphorothioate oligodeoxynucleotides: what is their origin and what is unique about them? Antisense Nucl. Acid Drug. Dev. 10, 117-121 (2000).

16. Wang, L. et al. DNA phosphorothioation is widespread and quantized in bacterial genomes. Proc. Natl Acad. Sci. USA 108, 2963-2968 (2011).

17. Xiong, W., Zhao, G., Yu, H. \& He, X. Interactions of Dnd proteins involved in bacterial DNA phosphorothioate modification. Front. Microbiol. 6, 1139 (2015).

18. He, W. et al. Regulation of DNA phosphorothioate modification in Salmonella enterica by DndB. Sci. Rep. 5, 12368 (2015).

19. Chen, C. et al. Convergence of DNA methylation and phosphorothioation epigenetics in bacterial genomes. Proc. Natl Acad. Sci. USA 114, 4501-4506 (2017).

20. Cao, B. et al. Pathological phenotypes and in vivo DNA cleavage by unrestrained activity of a phosphorothioate-based restriction system in Salmonella. Mol. Microbiol. 93, 776-785 (2014)

21. Snyder, J. C., Bolduc, B. \& Young, M. J. 40 Years of archaeal virology: Expanding viral diversity. Virology 479-480, 369-378 (2015).

22. Ackermann, H. -W. \& Prangishvili, D. Prokaryote viruses studied by electron microscopy. Arch. Virol. 157, 1843-1849 (2012).

23. Prangishvili, D. et al. The enigmatic archaeal virosphere. Nat. Rev. Microbiol. 15, 724-739 (2017)

24. Munson-McGee, J. H., Snyder, J. C. \& Young, M. J. Archaeal viruses from high-temperature environments. Genes (Basel) 9, pii: E128 (2018).

25. Krupovic, M., Cvirkaite-Krupovic, V., Iranzo, J., Prangishvili, D. \& Koonin, E. V. Viruses of archaea: Structural, functional, environmental and evolutionary genomics. Virus Res. 244, 181-193 (2018).

26. Roberts, R. J., Vincze, T., Posfai, J. \& Macelis, D. REBASE-a database for DNA restriction and modification: enzymes, genes and genomes. Nucl. Acids Res. 38, D234-D236 (2010).

27. Ishikawa, K. et al. Discovery of a novel restriction endonuclease by genome comparison and application of a wheat-germ-based cell-free translation assay: PabI (5'-GTA/C) from the hyperthermophilic archaeon Pyrococcus abyssi. Nucl. Acids Res. 33, e112 (2005).

28. Morgan, R., Xiao, J. \& Xu, S. Characterization of an extremely thermostable restriction enzyme, PspGI, from a Pyrococcus strain and cloning of the PspGI restriction-modification system in Escherichia coli. Appl. Environ. Microbiol. 64, 3669-3673 (1998).

29. Prangishvili, D. A., Vashakidze, R. P., Chelidze, M. G. \& Gabriadze, I. A restriction endonuclease SuaI from the thermoacidophilic archaebacterium Sulfolobus acidocaldarius. FEBS Lett. 192, 57-60 (1985).

30. Watanabe, M., Yuzawa, H., Handa, N. \& Kobayashi, I. Hyperthermophilic DNA methyltransferase M.PabI from the archaeon Pyrococcus abyssi. Appl. Environ. Microbiol. 72, 5367-5375 (2006).

31. Mattiroli, F. et al. Structure of histone-based chromatin in Archaea. Science 357, 609-612 (2017).

32. Cao, B. et al. Genomic mapping of phosphorothioates reveals partial modification of short consensus sequences. Nat. Commun. 5, 3951 (2014).

33. Flusberg, B. A. et al. Direct detection of DNA methylation during singlemolecule, real-time sequencing. Nat. Methods 7, 461-465 (2010).

34. Tong, T. et al. Occurrence, evolution, and functions of DNA phosphorothioate epigenetics in bacteria. Proc. Natl Acad. Sci. USA 115, E2988-E2996 (2018).

35. Selvy, P. E., Lavieri, R. R., Lindsley, C. W. \& Brown, H. A. Phospholipase D: Enzymology, functionality, and chemical modulation. Chem. Rev. 111, 6064-6119 (2011).

36. Snider, J., Thibault, G. \& Houry, W. A. The AAA + superfamily of functionally diverse proteins. Genome Biol. 9, 216 (2008).

37. Chen, F. et al. Crystal structure of the cysteine desulfurase DndA from Streptomyces lividans which is involved in DNA phosphorothioation. PLoS ONE 7, e36635 (2012)

38. Wang, Y. et al. Identification, characterization, and application of the replicon region of the halophilic temperate sphaerolipovirus SNJ1. J. Bacteriol. 198, 1952-1964 (2016).

39. Schwarz, F. W. et al. The helicase-like domains of type III restriction enzymes trigger long-range diffusion along DNA. Science 340, 353-356 (2013).
40. Murray, N. E. Type I restriction systems: sophisticated molecular machines (a legacy of Bertani and Weigle). Microbiol. Mol. Biol. Rev. 64, 412-434 (2000).

41. Zhao, Y., Stuckey, J. A., Lohse, D. L. \& Dixon, J. E. Expression, characterization, and crystallization of a member of the novel phospholipase D family of phosphodiesterases. Protein Sci. 6, 2655-2658 (1997).

42. Zaremba, M. et al. DNA cleavage by $C g l \mathrm{I}$ and $N g o A V I I$ requires interaction between $\mathrm{N}$ - and R-proteins and extensive nucleotide hydrolysis. Nucl. Acids Res. 42, 13887-13896 (2014).

43. Lagunavicius, A., Sasnauskas, G., Halford, S. E. \& Siksnys, V. The metalindependent type IIs restriction enzyme BfiI is a dimer that binds two DNA sites but has only one catalytic centre. J. Mol. Biol. 326, 1051-1064 (2003).

44. Hirano, T. At the heart of the chromosome: SMC proteins in action. Nat. Rev. Mol. Cell Biol. 7, 311-322 (2006).

45. Wang, Y., Chen, B., Sima, L., Cao, M. \& Chen, X. Construction of expression shuttle vectors for the Haloarchaeon natrinema sp. J7 based on its chromosomal origins of replication. Archaea 2017, 423707 (2017).

46. Breuert, S., Allers, T., Spohn, G. \& Soppa, J. Regulated polyploidy in halophilic archaea. PLoS ONE 1, e92 (2006)

47. Yamamoto, K. R., Alberts, B. M., Benzinger, R., Lawhorne, L. \& Treiber, G. Rapid bacteriophage sedimentation in the presence of polyethylene glycol and its application to large-scale virus purification. Virology 40, 734-744 (1970).

48. Bondy-Denomy, J., Pawluk, A., Maxwell, K. L. \& Davidson, A. R. Bacteriophage genes that inactivate the CRISPR/Cas bacterial immune system. Nature 493, 429 (2013).

49. Letunic, I. \& Bork, P. Interactive tree of life (iTOL) v3: an online tool for the display and annotation of phylogenetic and other trees. Nucl. Acids Res. 44, W242-W245 (2016).

50. Rice, P., Longden, I. \& Bleasby, A. EMBOSS: the European molecular biology open software suite. Trends Genet. 16, 276-277 (2000).

\section{Acknowledgements}

We sincerely thank Li Huang, Hua Xiang, Changsheng Zhang, Hao Bian and Qian Tan for their valuable suggestions and help with our experiments. We sincerely thank Dr. Suzanna Brauer for providing the DNA sample for $M$. boonei 6A8. This work was supported by grants from the National Science Foundation of China (31720103906, 31520103902 and 31670072). The work of Xiangdong Chen was supported by grants from the National Science Foundation of China (31570174).

\section{Author contributions}

L.W. and Shi Chen designed the research; L.X., S.L., Y.X., B.Z., Y.G., Y.Z. and B.C. conducted the experiments; L.X., S.L., Si Chen, Y.X., B.Z., Y.G., Y.Z., B.C., J.L., Z.D., X.C., L.W. and Shi Chen analysed the data; and L.X., L.W. and Shi Chen wrote the manuscript

\section{Additional information}

Supplementary Information accompanies this paper at https://doi.org/10.1038/s41467 019-09390-9.

Competing interests: The authors declare no competing interests.

Reprints and permission information is available online at http://npg.nature.com/ reprintsandpermissions/

Journal peer review information: Nature Communications thanks Chunyang Cao, Elisabeth Raleigh and the other anonymous reviewer for their contribution to the peer review of this work.

Publisher's note: Springer Nature remains neutral with regard to jurisdictional claims in published maps and institutional affiliations.

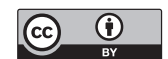

Open Access This article is licensed under a Creative Commons Attribution 4.0 International License, which permits use, sharing, adaptation, distribution and reproduction in any medium or format, as long as you give appropriate credit to the original author(s) and the source, provide a link to the Creative Commons license, and indicate if changes were made. The images or other third party material in this article are included in the article's Creative Commons license, unless indicated otherwise in a credit line to the material. If material is not included in the article's Creative Commons license and your intended use is not permitted by statutory regulation or exceeds the permitted use, you will need to obtain permission directly from the copyright holder. To view a copy of this license, visit http://creativecommons.org/ licenses/by/4.0\%

(C) The Author(s) 2019 